\title{
Vibration Electrochemical Micromachining Based on Coulostatic Analysis
}

\author{
Uk Su Kim, Yoon Jun Jung, and Jeong Woo Park
}

\begin{abstract}
One primary issue in pulse electrochemical micromachining is using pulses of electrical current to control precise machining resolution as well as the uniform electrolyte flow inside inter electrode gap between two electrodes. Periodical replacement of electrolyte flush away generated heat and gas bubbles which interrupt stable electrochemical reaction with uniform ionic charging in electrolyte. Though PECM require precise control of electrical parameters, such as pulse time, duty factor, applied current/voltage and total machining time, quantitative analysis of these parameter, especially pulse time, has not been introduced. This paper demonstrates rough prediction process of pulse time and machining resolution by analyzing high resolution pulse signals acquired from PECM operation. Additionally this research suggests vibration electrochemical polishing (VECP) assisted by ultrasonic vibration for enhancing electrochemical reaction and surface quality compared to the conventional ECP. The localized roughness of work material is measured by atomic force microscopy (AFM) for detailed information on surface. Besides roughness, overall surface quality and productivity etc. are compared with conventional ECP.
\end{abstract}

Index Terms-Electrochemical micro-machining, pulse electro chemical machining, vibration electrochemical polishing, coulostatic analysis.

\section{INTRODUCTION}

As various fields of industries develop nowadays, smaller equipment, hyperfine machining technology, anti-corrosion, and cleanliness become important. These issues have been improved in various ways with the developments of cutting edge devices, which could not be realized with the past technology. In the meantime, contact type processing methods involve problems in material strength/crack, thermal deformation, wear of tools, etc. To resolve these problems, contactless type special processing methods are being developed recently. Among them Electrochemical Polishing (ECP) technology is a representative contactless surface processing type, which allows polishing during the dissolution process of electrolyte on the electrode surface. Application of electrical current to the cathode in pulses, rather than continuous DC, offers significant improvements in dimensional accuracy as compared with conventional ECM. Basic theoretical work and industrial practice have

Manuscript received October 23, 2012; revised January 5, 2013.

Uk Su Kim is with the Department of Advanced Parts and Materials Engineering, Chosun University, 375, Seosuk-dong, Dong-gu Gwangju, 501-759, Korea (e-mail: uksunano@gmail.com).

Yoon Jun Jung and Jeong Woo Park are with the Department of Mechanical Design Engineering, Chosun university, 375, Seosuk-dong, Dong-gu Gwangju, 501-759, Korea (e-mail: yoonjun88@gmail.com, jwoopark@chosun.ac.kr). indicated that pulse electrochemical micromachining (PECM) offers considerable potential for enhancement of the ECM process. In PECM, periodic replacement of electrolyte in inter electrode gap makes it possible to apply a higher instant current density during the pulse time, leading to a significant improvement of surface quality. The smaller electrode gap results in an improved accuracy control [1], [2] While pulse electrochemical process enables the minimum inter electrode gap down to the micrometer scale and shows the possibilities of micromachining, the control of pulse parameters is on the rise. There have been some attempts to study the relation of pulse signals in electrochemical processes. K. P. Rajurkar group [3] and R. Schuster group [4] have studied about the rough relation of the pulse signals only to the inter electrode gap. Additionally Discharge machining, using ultrasonic vibration, there is research that facilitates the supply of the electrolyte between the tool and the workpiece. tact using ultrasound and during drilling, machining precision machined from improved practices. and Contact using ultrasound and during drilling, machining precision machined from improved practices. This study demonstrates a novel hybrid surface polishing process combining non-traditional electrochemical polishing(ECP) with external artificial ultrasonic vibration.

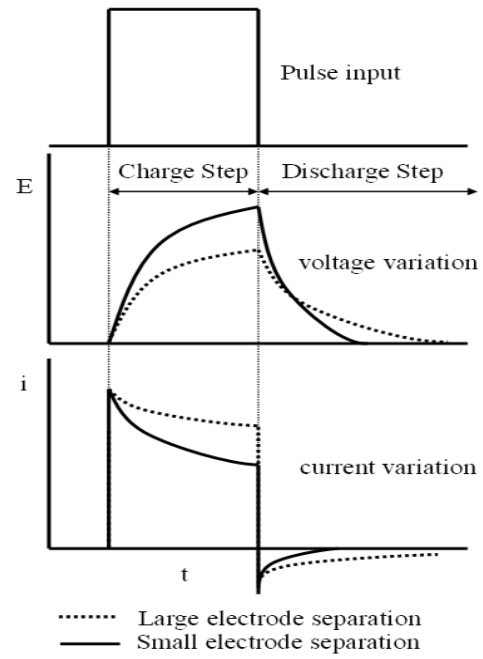

Fig. 1. Schematic diagram of voltage and current variations during the charge/discharge step of double layer in PECM cell. According to charging/discharging of electrical double layer, higher peak shows the tool electrode and work sample is positioned in close proximity.

\section{THEORETICAL BACKGROUND: ECP/PECP}

The concept of our process is mainly based on charging/discharging the electrical double layer in which the charge/discharge of an electrode stands face-to-face with an ionic charge in the solution This sequential charging and 
discharging of the double layer can be analyzed as a capacitor model when short voltage pulses are applied. Several studies have used an electrical double layer capacitor model in analyzing electrochemical process [5]. In PECM, an electrochemical cell can be remodeled by a simplified equivalent circuit of two electrodes immersed in electrolyte, whose resistance is proportional to the length of the current path; that is, the distance between tool electrode and work sample. The electrical double layer is composed of a compact double layer (CDL), which extends from the inner Helmholtz plane (IHP) to the outer Helmholtz plane (OHP), and a diffuse double layer (DDL), which covers the bulk electrolyte, as shown in Fig. 1 [6]. From capacitor analyses, a time constant $\tau$ can be defined as

$$
\tau=R C=\rho_{p} d c_{D L}
$$

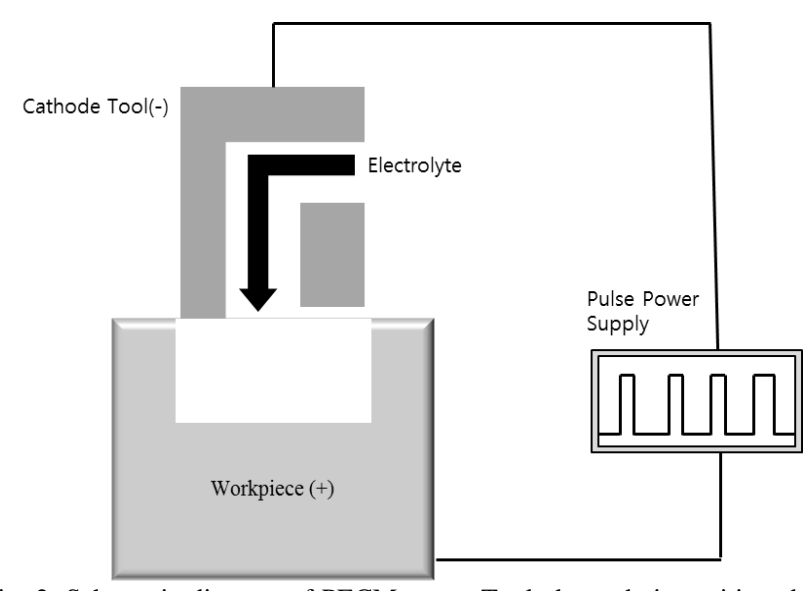

Fig. 2. Schematic diagram of PECM setup. Tool electrode is positioned in close proximity to the work sample and the electrolyte is supplied from outer apparatus. The in-process information of signal variations is recorded continuously by high resolution current probe.

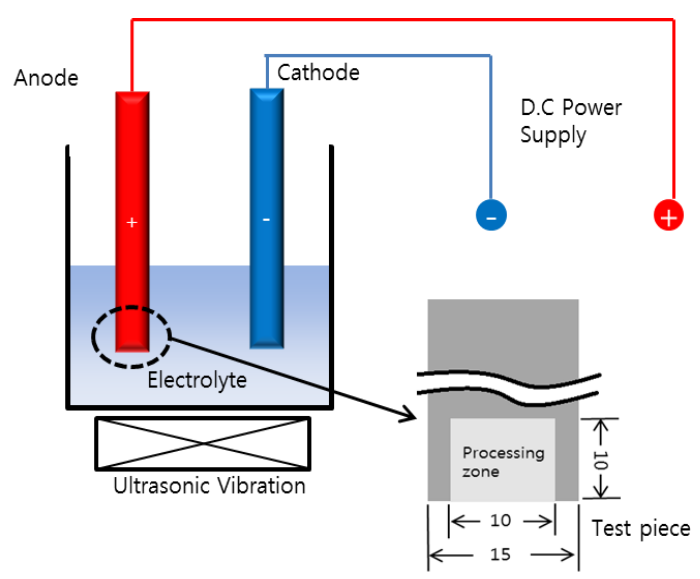

Fig. 3. Schematic diagram of ultrasonic ECP.

This time constant is a criterion for how fast the reaction occurs. The machining resolution or inter electrode gap $d$ is proportional to the time constant $\tau$ because the specific polarization resistance $\rho_{p}$, a factor defined by the electrolyte, and the electrical double layer capacity $c_{D L}$, defined by the summation of $c_{C D L}$ and $c_{D D L}$, are constant. The time constant for charging the electrochemical double layers on the electrodes is small enough for significant charging at electrode separation under millimeter range. Because the rates of electrochemical reactions are exponentially dependent on the potential drop in electrical double layer, the reactions are strongly confined to these polarized electrode regions in very close proximity. This methods contrast with conventional electroforming methods, in which the application of continuous DC voltage causes uniform electrical double layer charging and the reaction rate is mainly defined by the current density in the electrolyte, thus enabling only limited spatial resolution of about $0.1 \mathrm{~mm}$. [7] According to the charging/discharging of electrical double layer in electrochemical cell as illustrated in Fig. 1, the higher charging peak of the current and voltage indicates the narrow inter electrode gap.

\section{EXPERIMENTS}

Fig. 2 shows the schematic diagram of PECM. Pulse voltage from high frequency pulse generator is applied into the inter electrode gap between tool electrode and work sample, and the electrolyte solution is supplied from the external electrolyte supplier to make the electrochemical reaction homogeneous flushing away the generated gas and heat; it is circulated through a micro-filter to remove micro debris. The work sample is stainless steel 304 thickness of $30 \mu \mathrm{m}$ and $200 \mu \mathrm{m}$, the tool electrode is platinum $(\mathrm{Pt})$ wire with $75 \mu \mathrm{m}$-diameter. The sidewall of tool electrode is coated by insulating material and exposed flat top face is polished by mechanical finishing process. Fully programmable $50 \mathrm{MHz}$ pulse generator is HP8116A (Hewlett-Packard) with variable pulse width, $32 \mathrm{~V}$ peak to peak (into open circuit) maximum output amplitude. The high-speed data acquisition system is TDS 360 digital oscilloscope (Tektronix) with bandwidth of $200 \mathrm{MHz}$ and sampling rate of $500 \mathrm{MS} / \mathrm{s}$. Current prove to acquire the high frequency current pulses is CT-2 (Tektronix) with frequency response of $1.2 \mathrm{kHz}$ to $200 \mathrm{MHz}$, sensitivity of $1 \mathrm{mV} / \mathrm{mA} \pm 3 \%$ into a $50 \Omega$ load and pulse current rating of 36 A. This current prove is connected to a P6041 prove cable (Tektronix). A P6136 (Tektronix), voltage prove to acquire the high frequency voltage pulses, is applied in this system. Electrochemical polishing is a process that makes flat surface by dissolving the fine protruded parts of a workpiece in the electrolytic bath with an external source of electricity Fig. 1 is an outline drawing of electrochemical polishing by supersonic vibration used in this experiment. As shown in the outline drawing, this experiment consists of a power generating device $(50 \mathrm{~V}, 12 \mathrm{~A})$ and a supersonic vibration generation device $(40 \mathrm{kHz}, 150 \mathrm{~W})$. In the electrolytic bath, $2.4 \mathrm{M} \mathrm{H}_{2} \mathrm{SO}_{4}, 5.9 \mathrm{M} \mathrm{H}_{3} \mathrm{PO}_{4}$, and ultrapure water $\left(\mathrm{H}_{2} \mathrm{O}\right)$ are mixed in certain ratios. Stainless steel is used for anode. Insoluble and low electric resistant Copper $(\mathrm{Cu})$ is used for anode. In the electrochemical polishing process, insulating paste is applied to the whole area except a square of $10 \mathrm{~mm} \times$ $10 \mathrm{~mm}$, in order to process a certain area of stainless steel anode. In the process the distance between anode and cathode was $10 \mathrm{~mm}$ to connect the voltage of $7 \mathrm{~V}$ for 150 seconds Processed surface was measured locally with AFM (XE-100) of PISA Company. With the results surface shape affected by supersonic waves was compared and analyzed. These 
experimental systems for PECM are shown in Fig. 2. Fig. 3

\section{RESULT}

Fig. 4 show the results measured by AFM of original stainless steel surface before electrochemical polishing process. Measurement results revealed the roughness of the surface in mountainous shape was 27Ra. Fig. 5 shows the results of electrochemical polishing using a normal direct current. Ex-periment results indicated the improvement of surface roughness compared with the surface before the process. However it was also peak and valley in creased more densely. Fig. 6 shows the experiment results of vibration electro-chemical polishing when supersonic vibration (frequency $40 \mathrm{kHz}$, output $150 \mathrm{~W}$ ) was applied to the existing electrochem-ical polishing method). Experiment results of vibration elec-trochemical polishing revealed that the number of micro pit was reduced compared with the surface of the existing elec-trochemical polishing process, and surface roughness was also improved from $7 \mathrm{Ra}$ to $2.1 \mathrm{Ra}$. The machining resolutions depend on the time constants $\tau$ ( $d \approx \tau / \rho_{p} c_{D L}$ ) on the fixed electrolyte concentration. As PECM proceeds under proper pulse time and voltage conditions, the increase of ion charge within inter electrode gap leads to excessive dissolution of work sample, which makes machining resolution over theoretical prediction. After enough machining time, this excessive metallic dissolution will be confined due to increasing inter electrode gap, which leads machining resolution to approach theoretical prediction. From the linear correlation between time constant $\tau$, specific polarization resistance of electrolyte $\rho_{P}$, and spatial resolution $d$, it can be conjectured that shortening the pulse duration should linearly increase the machining precision. However, experimental apparatus should be equipped with higher resolution positioning stages with nanoscale step movement in proportional with the shortening pulse time. After enough machining time, this excessive metallic dissolution will be confined due to increasing inter electrode gap, which leads machining resolution to approach theoretical prediction. From the linear correlation between time constant $\tau$, specific polarization resistance of electrolyte $\rho_{P}$, and spatial resolution $d$, it can be conjectured that shortening the pulse duration should linearly increase the machining precision. However, experimental apparatus should be equipped with higher resolution positioning stages with nanoscale step movement in proportional with the shortening pulse time. Fig 8 shows an attempt to fabricate predefined pattern with uniform 9 11 $\mu \mathrm{m}$ depth on STS304 surface for producing non-contact hydrodynamic bearing for small electronics application by PECM process. PECM contrasts with conventional electrochemical methods, in which the application of continuous DC voltage causes uniform electrical double layer charging and the electrochemical reaction rate is mainly defined by the current density in the electrolyte, thus enabling only limited spatial resolution as mentioned above.

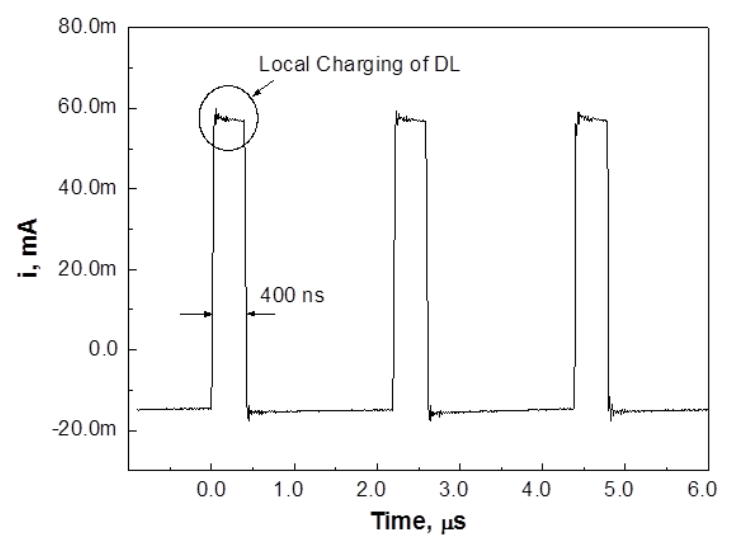

Fig. 4. Series of current pulses ( $455 \mathrm{kHz}$ sequence of $400 \mathrm{~ns}, 9.0 \mathrm{~V})$

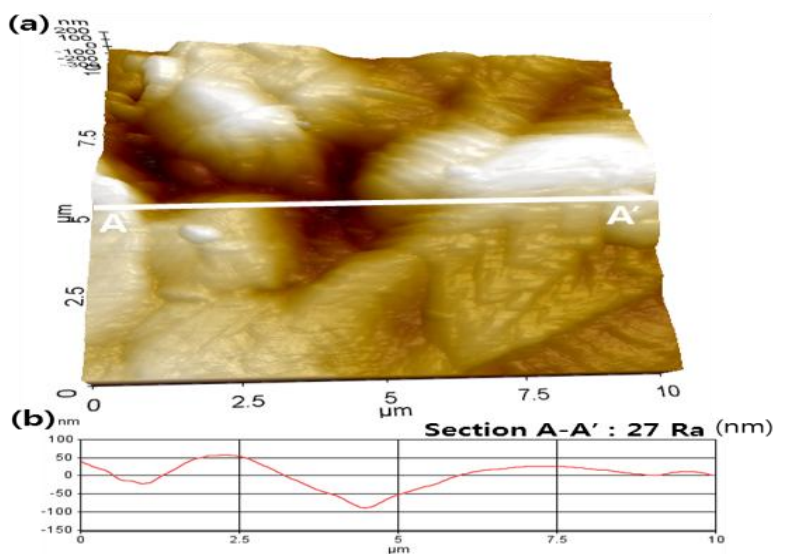

Fig. 5. Sample surface before ECP. (a) AFM topographical image, (b) cross sectional profile along $\mathrm{AA}^{\prime}$ in (a)

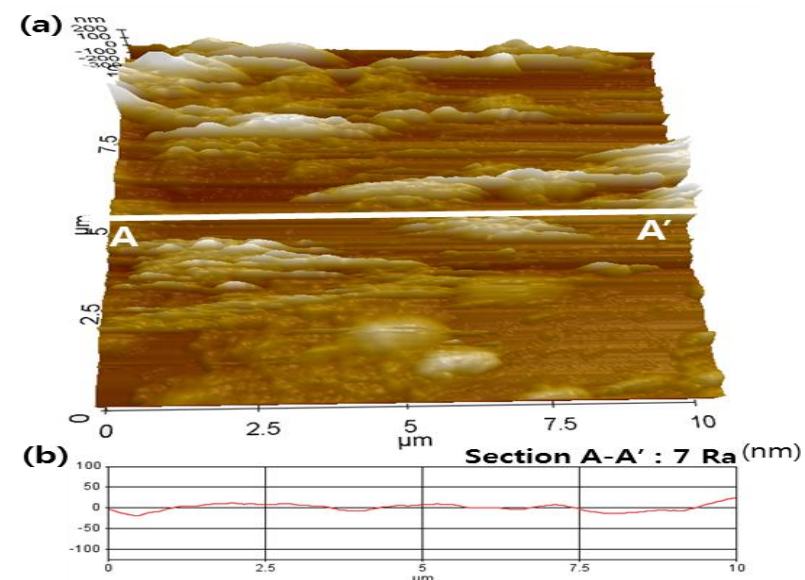

Fig. 6. Sample surface after ECP. (a) AFM topographical image, (b) cross sectional profile along AA' in (a).

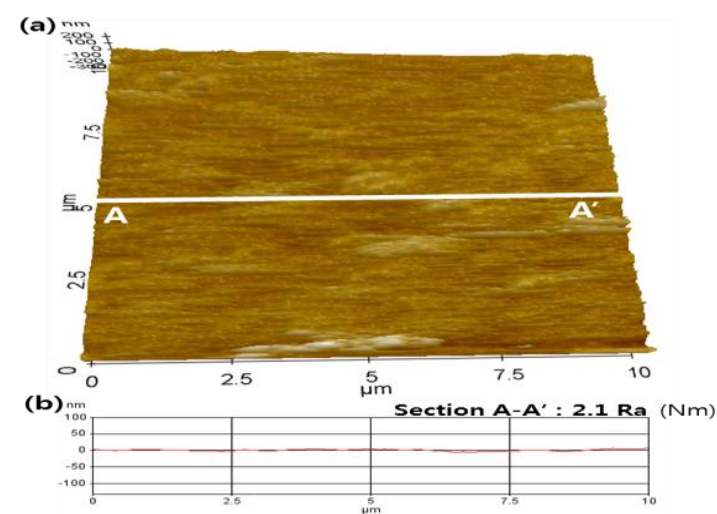

Fig. 7. Sample surface ultrasonic vibration ECP. (a) AFM topographical image, (b) cross sectional profile along $\mathrm{AA}^{\prime}$ in (a). 


\section{CONCLUSIONS}

This study also differs from recent methods using the known specific electrolyte resistances, in which one cannot analyze the spatial resolution theoretically because of unknown properties of the electrolyte though he discovers novel mixed electrolytes. Hence there are only limited choices of electrolyte in case of analyzing the PECM process. The unique of this study is introducing a possible novel way to specify the specific electrolyte resistance by analyzing ultra short current signals based on coulostatic relaxation processes. Additionally Through the experiment, this paper could identify hybrid type vibration electrochemical polish process adopting supersonic wave, compared with the existing electrochemical polishing process, induced lower frequency of the peak and valley on the processed surface, and I could check more flat surface and improved roughness. With the improved cleanliness and detergency identified by this experiment results, this technology can be applied to various industrial fields like bio and medical areas.

\section{ACKNOWLEDGMENT}

This research was supported by Basic Science Research Program through the National Research Foundation of Korea (NRF) funded by the Ministry of Education, Science and Technology (2012R1A1B4004235).

\section{REFERENCES}

[1] E. S. Lee, J. W. Park, and Y. H. Moon, "Development of Ultra Clean Machining Technology with Electrolytic Polishing Process," International Journal of the Korean Society of Precision Engineering, vol. 2, pp. 18-25, 2001

[2] K. Chikamori, "Possibilities of Electro-chemical Micromachining," International Journal of JSPE, vol. 32, pp. 37-38, 1998.

[3] K. P. Rajurkar, B. Wei, and J. Kozak, "Study of Pulse Electrochemical Machining Characteristics," CIRP Annals - Manufacturing Technology, vol. 42, pp. 231-234, 1993

[4] R. Schuster and V. Kirchner, "Electrochemical Micromachining," Science, vol. 289, pp. 98-101, 2000.

[5] R. H. Baughman, C. Cui, and M. Kertesz, "Carbon nanotube actuators," Science, vol. 284, pp. 1340-1344, 1999.

[6] Y. B. Kim and J. W. Park, "Corrosion Rate Evaluation of Pulse Electrochemical Polishing for Stainless Steel," Adv. Sci. Lett., vol. 14, pp. 227-230, 2012

[7] J. W. Park and D. W. Lee, "Pulse Electrochemical Polishing for Microrecesses based on a Coulostatic Analysis," International Journal of Advanced Manufacturing Technology, vol. 40, pp. 742-748, 2009.

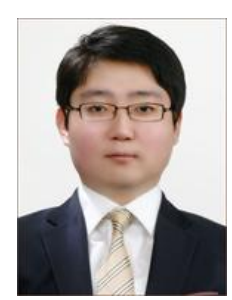

Uksu. Kim was born on December 8, 1986 in Gwangju, Korea. $\mathrm{He}$ is attending the Chosun university. He was graduated the Department of Mechanical Design Engineering in Chosun University. Uk Su Kim is a Master's course student at the Department of Advanced Parts and Materials Engineering. And he worked in the ultra-precision machining laboratory from in 2012. Mr. Uksu. Kim is in the department of advanced parts and materials engineering, Chosun University, in Korea. Mr. Youngbin. Kim is in the department of advanced parts and materials engineering, Chosun University, in Korea. Prof. Jeongwoo. Park is in the department of mechanical design engineering, Chosun University, in Korea. 